\title{
Normative Data for the Balance Error Scoring System in Adults
}

\author{
Grant L. Iverson ${ }^{1,2,3}$ and Michael S. Koehle $e^{3,4}$ \\ ${ }^{1}$ Department of Physical Medicine and Rehabilitation, Harvard Medical School, Boston, MA, USA \\ ${ }^{2}$ Red Sox Foundation and Massachusetts General Hospital Home Base Program, Boston, MA, USA \\ ${ }^{3}$ Copeman Healthcare Centre, Vancouver, BC, Canada V6Z 2L4 \\ ${ }^{4}$ School of Kinesiology and Division of Sport Medicine, University of British Columbia, 210-6081 University Boulevard, \\ Vancouver, BC, Canada V6T 1Z1
}

Correspondence should be addressed to Michael S. Koehle; michael.koehle@ubc.ca

Received 11 August 2012; Accepted 18 February 2013

Academic Editor: Francois Prince

Copyright (C) 2013 G. L. Iverson and M. S. Koehle. This is an open access article distributed under the Creative Commons Attribution License, which permits unrestricted use, distribution, and reproduction in any medium, provided the original work is properly cited.

Background. The balance error scoring system (BESS) is a brief, easily administered test of static balance. The purpose of this study is to develop normative data for this test. Study Design. Cross-sectional, descriptive, and cohort design. Methods. The sample was drawn from a population of clients taking part in a comprehensive preventive health screen at a multidisciplinary healthcare center. Community-dwelling adults aged 20-69 $(N=1,236)$ were administered the BESS within the context of a fitness evaluation. They did not have significant medical, neurological, or lower extremity problems that might have an adverse effect on balance. Results. There was a significant positive correlation between BESS scores and age $(r=.34)$. BESS performance was similar for participants between the ages of 20 and 49 and significantly declined between ages 50 and 69. Men performed slightly better than women on the BESS. Women who were overweight performed significantly more poorly on the test compared to women who were not overweight $(P<.0001$; Cohen's $d=.62)$. The BESS normative data are stratified by age and sex. Conclusions. These normative data provide a frame of reference for interpreting BESS performance in adults who sustain traumatic brain injuries and adults with diverse neurological or vestibular problems.

\section{Introduction}

Problems with balance and postural stability can arise from injuries or diseases affecting the vestibular system and/or brain. Examples include stroke [1], Parkinson's disease [2], multiple sclerosis [3], traumatic brain injury [4-10], blast exposure in the military [11], and sport-related concussion [12]. Blunt trauma to the head can cause persistent problems with dizziness and balance via a variety of vestibular system problems such as labyrinthine concussion, rupture of the round window membrane, delayed endolymphatic hydrops, and benign paroxysmal positional vertigo due to canalolithiasis [13].

The balance error scoring system (BESS; $[10,14-16])$ is a rapid, relatively easy-to-administer, and inexpensive measure of static balance and postural stability. A combination of three stances (narrow double leg stance, single leg stance, and tandem stance) and footing surfaces (firm surface/floor or medium density foam) is used for the test. Iverson et al. [17] presented preliminary normative data for the BESS derived from community-dwelling adults $(N=589)$ between the ages of 20 and 69 . The purpose of this study is to expand the normative reference data for this test for adults and older adults.

\section{Methods}

2.1. Participants. Community-dwelling adults $(N=1,236)$ were administered the BESS within the context of a comprehensive preventive health screen at a multidisciplinary healthcare center. This sample included the original 589 subjects from Iverson et al. [17]. The average age of the sample was 49.5 years $(S D=10.8$; range $=20-69)$. There were 739 men $(59.8 \%)$ and $497(40.2 \%)$ women. The average body mass index $(\mathrm{BMI})$ for men was $28.1(\mathrm{SD}=4.1$, range $=16.2-49.0)$ 


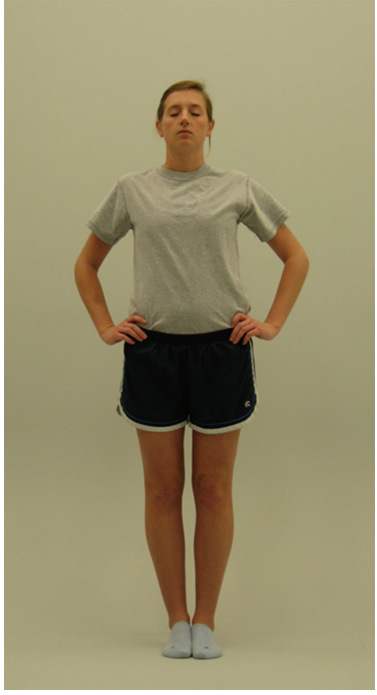

(a)

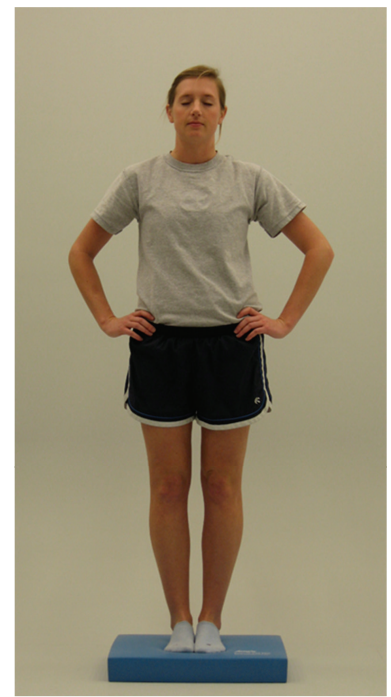

(d)

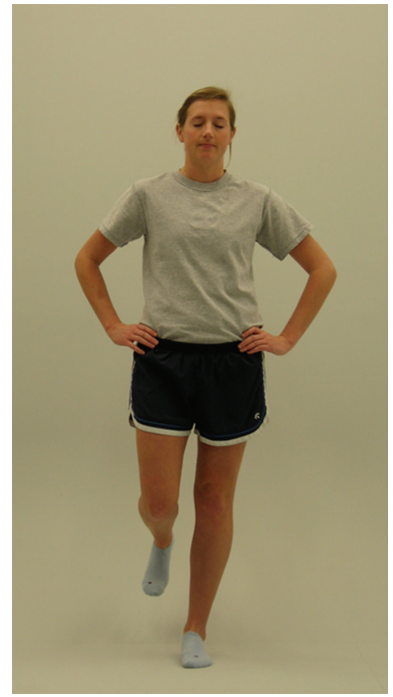

(b)

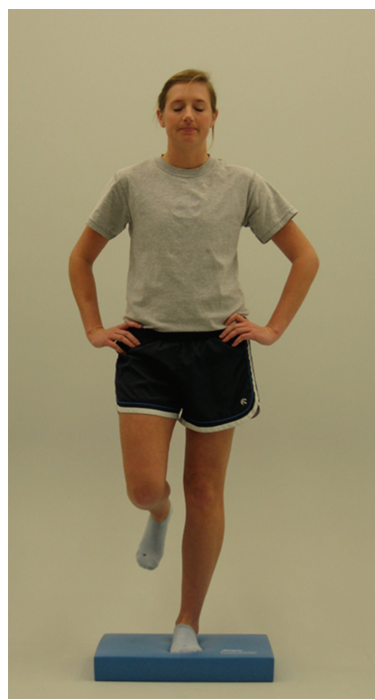

(e)

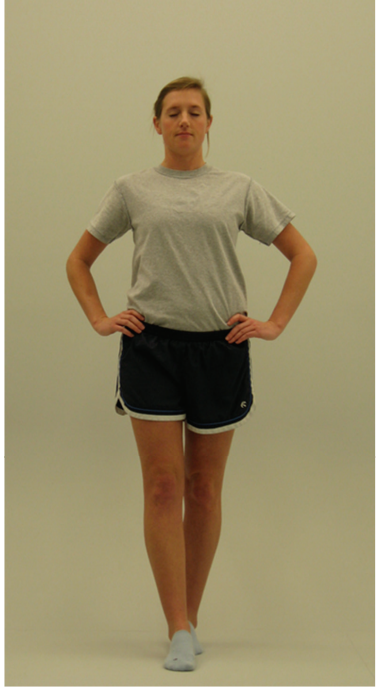

(c)

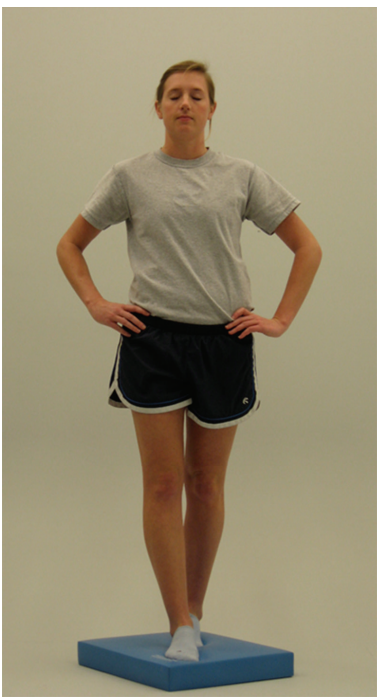

(f)

FIGURE 1: Balance error scoring system (BESS) performed on firm surface ((a)-(c)) and foam surface ((d)-(f)). Figure reprinted with permission from Davis et al. [24].

and for the women was $24.5(\mathrm{SD}=4.5$, range $=15.9-45.8)$. All medical charts were reviewed by a medical student and physician to exclude individuals with significant neurological, medical, or lower extremity conditions that could potentially have affected their balance.

\subsection{Measure. The Balance Error Scoring System (BESS;} $[10,14-16]$ ) uses a combination of three stances (narrow double leg stance, single leg stance, and tandem stance) and two footing surfaces (firm surface/floor or medium density foam). The first 3 stances, on a hard surface, comprise the test. Each stance is held, with hands on hips and eyes closed, for 20 seconds. "Error" points are given for specific behaviors, including opening eyes, lifting hands off hips, or stepping, stumbling, or falling. Therefore, a higher total score reflects worse performance on the test. All tests were scored by professional kinesiologists trained in the administration of the BESS and who perform the test daily as part of their practice. For reliability, these kinesiologists evaluated BESS performance from a video with an ICC $=0.88$. BESS performance can vary, or be influenced by, a number of factors including the type of sport played [18], a history of ankle injuries and ankle instability [19], and exertion and fatigue $[20,21]$. Uninjured athletes can have a subtle learning effect on the BESS when it is administered over brief retest intervals $[22,23]$. The BESS is illustrated in Figure 1.

\section{Results}

There was a small significant positive correlation between BESS scores and age $(r=.34, P<.0001)$. Comparing the six 
TABLE 1: Normative reference values for the BESS stratified by age.

\begin{tabular}{|c|c|c|c|c|c|c|c|c|c|c|}
\hline Age & $N$ & Mean & Median & $\mathrm{SD}$ & Superior & Above average & Broadly normal & Below average & Poor & Very poor \\
\hline $20-29$ & 65 & 11.3 & 11.0 & 4.8 & $0-5$ & $6-7$ & $8-14$ & $15-17$ & $18-23$ & $24+$ \\
\hline $30-39$ & 173 & 11.5 & 11.0 & 5.5 & $0-4$ & $5-7$ & $8-15$ & $16-18$ & $19-26$ & $27+$ \\
\hline $40-49$ & 352 & 12.5 & 11.5 & 6.2 & $0-5$ & $6-8$ & $9-16$ & $17-20$ & $21-28$ & $29+$ \\
\hline $50-54$ & 224 & 14.2 & 12.0 & 7.5 & $0-6$ & $7-8$ & $9-18$ & $19-24$ & $25-33$ & $34+$ \\
\hline $55-59$ & 197 & 16.5 & 15.0 & 7.6 & $0-7$ & $8-10$ & $11-20$ & $21-28$ & $29-35$ & $36+$ \\
\hline $60-64$ & 148 & 18.0 & 16.5 & 7.8 & $0-8$ & $9-12$ & $13-22$ & $23-28$ & $29-40$ & $41+$ \\
\hline $65-69$ & 77 & 19.9 & 18.0 & 7.1 & $0-12$ & $13-15$ & $16-24$ & $25-32$ & $33-38$ & $39+$ \\
\hline \multicolumn{11}{|l|}{ Men } \\
\hline $20-29$ & $26^{*}$ & 10.4 & 10.0 & 4.4 & $0-4$ & $5-6$ & $7-14$ & 15 & $16-21$ & $22+$ \\
\hline $30-39$ & 97 & 11.5 & 11.0 & 5.5 & $0-4$ & $5-6$ & $7-15$ & $16-18$ & $19-26$ & $27+$ \\
\hline $40-49$ & 212 & 12.4 & 12.0 & 5.7 & $0-5$ & $6-7$ & $8-16$ & $17-20$ & $21-27$ & $28+$ \\
\hline $50-54$ & 142 & 13.6 & 12.0 & 6.9 & $0-6$ & 7 & $8-17$ & $18-23$ & $24-28$ & $29+$ \\
\hline $55-59$ & 117 & 16.4 & 15.0 & 7.2 & $0-7$ & $8-10$ & $11-20$ & $21-28$ & $29-34$ & $35+$ \\
\hline $60-64$ & 89 & 17.2 & 16.0 & 7.1 & $0-8$ & $9-11$ & $12-21$ & $22-27$ & $28-35$ & $36+$ \\
\hline $65-69$ & 56 & 20.0 & 18.0 & 7.3 & $0-12$ & $13-14$ & $15-23$ & $24-33$ & $34-39$ & $40+$ \\
\hline \multicolumn{11}{|c|}{ Women } \\
\hline $20-29$ & $39^{*}$ & 11.9 & 11.0 & 5.1 & $0-5$ & $6-7$ & $8-14$ & $15-19$ & $20-25$ & $26+$ \\
\hline $30-39$ & 76 & 11.4 & 10.5 & 5.6 & $0-4$ & $5-6$ & $7-15$ & $16-19$ & $20-27$ & $28+$ \\
\hline $40-49$ & 140 & 12.7 & 11.0 & 6.9 & $0-5$ & $6-7$ & $8-15$ & $16-20$ & $21-29$ & $30+$ \\
\hline $50-54$ & 82 & 15.1 & 13.0 & 8.2 & $0-7$ & $8-9$ & $10-20$ & $21-24$ & $25-35$ & $36+$ \\
\hline $55-59$ & 80 & 16.7 & 15.0 & 8.2 & $0-8$ & $9-10$ & $11-21$ & $22-28$ & 29-39 & $40+$ \\
\hline $60-64$ & 59 & 19.3 & 17.0 & 8.8 & $0-9$ & $10-12$ & $13-22$ & $23-31$ & $32-43$ & $44+$ \\
\hline $65-69$ & $21^{*}$ & 19.9 & 18.0 & 6.6 & $0-13$ & 14 & $15-24$ & $25-27$ & $28-38$ & $39+$ \\
\hline \multicolumn{11}{|c|}{ Women: $\mathrm{BMI} \geq 30$} \\
\hline $20-49$ & $27^{*}$ & 17.3 & 16.0 & 6.5 & $0-8$ & $9-12$ & $13-22$ & $23-27$ & $28-33$ & $34+$ \\
\hline $50-64$ & $32^{*}$ & 21.6 & 20.0 & 8.4 & $0-11$ & $12-14$ & $15-27$ & $28-32$ & $33-41$ & $42+$ \\
\hline
\end{tabular}

* Unusually small sample sizes limit the usefulness of these normative reference values. The maximum score for each trial was truncated at 10 points. BMI: body mass index. Body mass had a greater effect on balance performance in women than in men. Superior scores occur in fewer than $10 \%$ of the sample. Above average scores occur in approximately $15 \%$, broadly normal scores occur in approximately $50 \%$, below average scores occur in approximately $15 \%$, poor scores occur in approximately $8 \%$, and very poor scores occur in fewer than $2 \%$. These classification ranges correspond to the following percentile ranks: Very poor $<2$ nd percentile; poor $=2$ nd-9th percentile; below average $=10$ th -24 th percentile; broadly normal $=25$ th -75 th percentile; above average $=76$ th -90 th percentile; superior $>90$ th percentile.

age groups in Table 1, parametric and nonparametric analyses revealed an overall main effect for age $(F(6,1,235)=30.6, P<$ .0001 ; Levene's test for homogeneity of variances, $P<.0001$; Kruskal-Wallis $(6,1,236)=170.5, P<.0001$; IndependentSamples Median Test $(6,1,236)=128.8, P<.0001)$. BESS performance was similar for participants between the ages of 20 and 49 and significantly declined between ages 50 and 69. ANCOVA revealed slightly better BESS performance in men than in women $(F(2,1,235)=5.4, P<.021$, partial eta squared $=.004)$. The effect size for this difference was very small, however. There was a nonsignificant trend for men who were overweight $(\mathrm{BMI} \geq 30)$ to perform more poorly on the $\operatorname{BESS}(t(1,736)=1.92, P<.055, d=.16)$. Women who were overweight performed significantly more poorly on the test $(t(1,494)=4.33, P<.0001 ; d=.62)$. The BESS normative data are provided in Table 1.

\section{Discussion}

Balance can be adversely affected by a wide range of vestibular or neurological illnesses, injuries, or conditions. Moreover, age-associated declines in computerized dynamic posturography are well documented in the literature $[25,26]$. As seen in Table 1, balance and postural stability, as measured by the BESS, decline with age.

The BESS is a standardized, rapid, inexpensive, and screening test of postural stability that can be helpful for documenting deficits, monitoring recovery from injury, or tracking deterioration due to a neurological condition. It has been used in many studies with healthy athletes [18, 20$22,27,28]$, and as an outcome measure relating to ankle instability $[19,29,30]$ or sport-related concussion $[10,14,15]$. A systematic review of the BESS, published in 2011, provides important information regarding its reliability and validity [31]. The normative data in the present study provide a frame of reference for interpreting BESS performance across the lifespan in healthy adults. These normative reference values allow the clinician to classify a person's balance across a broad range, from superior (top 10\%) to very poor (bottom $2 \%$ ).

The clinical use of the normative reference values in Table 1 is straightforward. A person's total score can be classified in certain ranges relative to the normative reference 
values. For example, a 52-year-old man with a score of 20 would be classified as having "below average" balance relative to his age (i.e., men and women combined) and relative to men his age. His score is in the lower $25 \%$ of the normative subjects. A 45-year-old overweight woman who sustained a moderate traumatic brain injury three months prior to testing obtained a score of 25. Compared to women of her age, her balance was "poor" (i.e., lower 10\% of the normative subjects). Compared to a small sample of women who are overweight, her performance was below average (i.e., lower $25 \%$ of the normative sample). A 62 -year-old man participating in a cardiac rehabilitation program following a myocardial infarction obtained a score of 7 . Compared to men of his age, this score is in the "superior" range (i.e., upper $10 \%$ of the normative subjects). If a patient's balance is impaired, or the clinician decides that it might not be safe or necessary to test balance on the foam mat, then normative reference values for the Modified Balance Error Scoring System (M-BESS; first 3 stances on hard surface) can be used $[32,33]$. At present, there are no published studies related to the test-retest reliability of the BESS in adults (with the exception of athletes) or older adults, so it is not possible to estimate test-retest measurement error. Therefore, there is no statistical approach available for interpreting change in BESS scores across time in rehabilitation settings. Rehabilitation professionals using the BESS must simply rely on clinical judgment for interpreting change scores until future reliable change data becomes available.

When using these normative data, clinicians should keep in mind that they were derived from a single laboratory in Vancouver, Canada. This was a reasonably healthy adult sample that underwent a fitness evaluation at a private healthcare center in Canada. The individuals in this sample have a higher than average socioeconomic status (SES), and a relatively small percentage of them were obese, so the results might not generalize across the full spectrum of SES or to adults who are overweight.

Computerized dynamic posturography may reveal abnormalities in postural responses to changing sensory conditions that are not detected by measures such as the BESS [34, 35]. Moreover, balance problems can be more apparent under conditions of physical exertion in some patients [36]. Therefore, in rehabilitation settings, it is important to monitor balance in the context of progressively increasing levels of physical exertion.

\section{Conflict of Interests}

The authors declare no conflict of interests.

\section{Acknowledgments}

The authors thank Dr. Rebecca Glassford, Michael Greene, Hedieh Hafizi, Spencer Holowachuk, Frances Jones, Michelle Kaarto, Lisa Marescaux, and Nadine Sinnen for collecting the data used in this study, Boaz Saffer for assistance with the literature review, and Jennifer Bernardo for assistance with manuscript preparation. A portion of these data were presented at the annual conference of the American College of Rehabilitation Medicine, Atlanta, GA, USA, October 11-15, 2011.

\section{References}

[1] C. L. Pollock, J. J. Eng, and S. J. Garland, "Clinical measurement of walking balance in people post stroke: a systematic review," Clinical Rehabilitation, vol. 25, no. 8, pp. 693-708, 2011.

[2] N. E. Allen, C. Sherrington, S. S. Paul, and C. G. Canning, "Balance and falls in Parkinson's disease: a meta-analysis of the effect of exercise and motor training," Movement Disorders, vol. 26, no. 9, pp. 1605-1615, 2011.

[3] M. H. Cameron and S. Lord, "Postural control in multiple sclerosis: implications for fall prevention," Current Neurology and Neuroscience Reports, vol. 10, no. 5, pp. 407-412, 2010.

[4] B. D. Greenwald, D. X. Cifu, J. H. Marwitz et al., "Factors associated with balance deficits on admission to rehabilitation after traumatic brain injury: a multicenter analysis," Journal of Head Trauma Rehabilitation, vol. 16, no. 3, pp. 238-252, 2001.

[5] M. B. Rinne, M. E. Pasanen, M. V. Vartiainen, T. M. Lehto, J. M. Sarajuuri, and H. T. Alaranta, "Motor performance in physically well-recovered men with traumatic brain injury," Journal of Rehabilitation Medicine, vol. 38, no. 4, pp. 224-229, 2006.

[6] K. R. Kaufman, R. H. Brey, L. S. Chou, A. Rabatin, A. W. Brown, and J. R. Basford, "Comparison of subjective and objective measurements of balance disorders following traumatic brain injury," Medical Engineering and Physics, vol. 28, no. 3, pp. 234239, 2006.

[7] M. Campbell and A. Parry, "Balance disorder and traumatic brain injury: preliminary findings of a multi-factorial observational study," Brain Injury, vol. 19, no. 13, pp. 1095-1104, 2005.

[8] A. C. H. Geurts, G. M. Ribbers, J. A. Knoop, and J. van Limbeek, "Identification of static and dynamic postural instability following traumatic brain injury," Archives of Physical Medicine and Rehabilitation, vol. 77, no. 7, pp. 639-644, 1996.

[9] I. Gagnon, R. Forget, S. J. Sullivan, and D. Friedman, "Motor performance following a mild traumatic brain injury in children: an exploratory study," Brain Injury, vol. 12, no. 10, pp. 843853, 1998.

[10] M. McCrea, K. M. Guskiewicz, S. W. Marshall et al., "Acute effects and recovery time following concussion in collegiate football players: the NCAA concussion study," The Journal of the American Medical Association, vol. 290, no. 19, pp. 2556-2563, 2003.

[11] F. W. Akin and O. D. Murnane, "Head injury and blast exposure: vestibular consequences," Otolaryngologic Clinics of North America, vol. 44, no. 2, pp. 323-334, 2011.

[12] S. P. Broglio and T. W. Puetz, "The effect of sport concussion on neurocognitive function, self-report symptoms and postural control: a meta-analysis," Sports Medicine, vol. 38, no. 1, pp. 5367, 2008

[13] A. Ernst, D. Basta, R. O. Seidl, I. Todt, H. Scherer, and A. Clarke, "Management of posttraumatic vertigo," Otolaryngology-Head and Neck Surgery, vol. 132, no. 4, pp. 554-558, 2005.

[14] K. M. Guskiewicz, S. E. Ross, and S. W. Marshall, "Postural stability and neuropsychological deficits after concussion in collegiate athletes," Journal of Athletic Training, vol. 36, no. 3, pp. 263-273, 2001.

[15] B. L. Riemann and K. M. Guskiewicz, "Effects of mild head injury on postural stability as measured through clinical balance 
testing," Journal of Athletic Training, vol. 35, no. 1, pp. 19-25, 2000.

[16] K. M. Guskiewicz, "Postural stability assessment following concussion: one piece of the puzzle," Clinical Journal of Sport Medicine, vol. 11, no. 3, pp. 182-189, 2001.

[17] G. L. Iverson, M. L. Kaarto, and M. S. Koehle, "Normative data for the balance error scoring system: implications for brain injury evaluations," Brain Injury, vol. 22, no. 2, pp. 147-152, 2008.

[18] E. Bressel, J. C. Yonker, J. Kras, and E. M. Heath, "Comparison of static and dynamic balance in female collegiate soccer, basketball, and gymnastics athletes," Journal of Athletic Training, vol. 42, no. 1, pp. 42-46, 2007.

[19] C. L. Docherty, T. C. V. McLeod, and S. J. Shultz, "Postural control deficits in participants with functional ankle instability as measured by the balance error scoring system," Clinical Journal of Sport Medicine, vol. 16, no. 3, pp. 203-208, 2006.

[20] T. W. Susco, T. C. V. McLeod, B. M. Gansneder, and S. J. Shultz, "Balance recovers within 20 minutes after exertion as measured by the balance error scoring system," Journal of Athletic Training, vol. 39, no. 3, pp. 241-246, 2004.

[21] J. C. Wilkins, T. C. V. McLeod, D. H. Perrin, and B. M. Gansneder, "Performance on the balance error scoring system decreases after fatigue," Journal of Athletic Training, vol. 39, no. 2, pp. 156-161, 2004.

[22] T. C. V. McLeod, D. H. Perrin, K. M. Guskiewicz, S. J. Shultz, R. Diamond, and B. M. Gansneder, "Serial administration of clinical concussion assessments and learning effects in healthy young athletes," Clinical Journal of Sport Medicine, vol. 14, no. 5, pp. 287-295, 2004.

[23] T. C. Valovich, D. H. Perrin, and B. M. Gansneder, "Repeat administration elicits a practice effect with the balance error scoring system but not with the standardized assessment of concussion in high school athletes," Journal of Athletic Training, vol. 38, no. 1, pp. 51-56, 2003.

[24] G. A. Davis, G. L. Iverson, K. M. Guskiewicz, A. Ptito, and K. M. Johnston, "Contributions of neuroimaging, balance testing, electrophysiology, and blood markers to the assessment of sport-related concussion," The British Journal of Sports Medicine, vol. 43, supplement 1, pp. i36-i45, 2009.

[25] H. Cohen, L. G. Heaton, S. L. Congdon, and H. A. Jenkins, "Changes in sensory organization test scores with age," Age and Ageing, vol. 25, no. 1, pp. 39-44, 1996.

[26] M. Y. Liaw, C. L. Chen, Y. C. Pei, C. P. Leong, and Y. C. Lau, "Comparison of the static and dynamic balance performance in young, middle-aged, and elderly healthy people," Chang Gung Medical Journal, vol. 32, no. 3, pp. 297-304, 2009.

[27] Z. G. Fox, J. P. Mihalik, J. T. Blackburn, C. L. Battaglini, and K. M. Guskiewicz, "Return of postural control to baseline after anaerobic and aerobic exercise protocols," Journal of Athletic Training, vol. 43, no. 5, pp. 456-463, 2008.

[28] A. V. Patel, J. P. Mihalik, A. J. Notebaert, K. M. Guskiewicz, and W. E. Prentice, "Neuropsychological performance, postural stability, and symptoms after dehydration," Journal of Athletic Training, vol. 42, no. 1, pp. 66-75, 2007.

[29] M. Vikram, K. Sundaraganesh, M. Justine, M. Kurup, and J. H. Leonard, "Evaluation of postural control impairment using balance error scoring system among athletes with ankle injury: an effective tool in daily clinical practice," La Clinica Terapeutica, vol. 163, no. 5, pp. 383-386, 2012.

[30] J. R. Beazell, T. L. Grindstaff, L. D. Sauer, E. M. Magrum, C. D. Ingersoll, and J. Hertel, "Effects of a proximal or distal tibiofibular joint manipulation on ankle range of motion and functional outcomes in individuals with chronic ankle instability," Journal of Orthopaedic and Sports Physical Therapy, vol. 42, no. 2, pp. 125-134, 2012.

[31] D. R. Bell, K. M. Guskiewicz, M. A. Clark, and D. A. Padua, "Systematic review of the balance error scoring system," Sports Health, vol. 3, no. 3, pp. 287-295, 2011.

[32] G. L. Iverson and M. S. Koehle, "Normative data for the balance error scoring system in community-dwelling adults," Archives of Physical Medicine and Rehabilitation, vol. 92, pp. 1695-1696, 2011.

[33] G. L. Iverson and M. S. Koehle, "Normative data for the balance error scoring system in community-dwelling adults," in Proceedings of the American Congress of Rehabilitation Medicine, Atlanta, Ga, USA, 2011, October.

[34] F. O. Black, "What can posturography tell us about vestibular function?" Annals of the New York Academy of Sciences, vol. 942, pp. 446-464, 2001.

[35] J. E. Visser, M. G. Carpenter, H. van der Kooij, and B. R. Bloem, "The clinical utility of posturography," Clinical Neurophysiology, vol. 119, no. 11, pp. 2424-2436, 2008.

[36] T. Carver, S. Nadeau, and A. Leroux, "Relation between physical exertion and postural stability in hemiparetic participants secondary to stroke," Gait and Posture, vol. 33, no. 4, pp. 615619, 2011. 


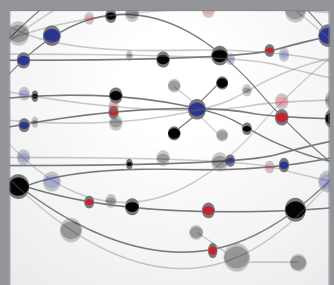

The Scientific World Journal
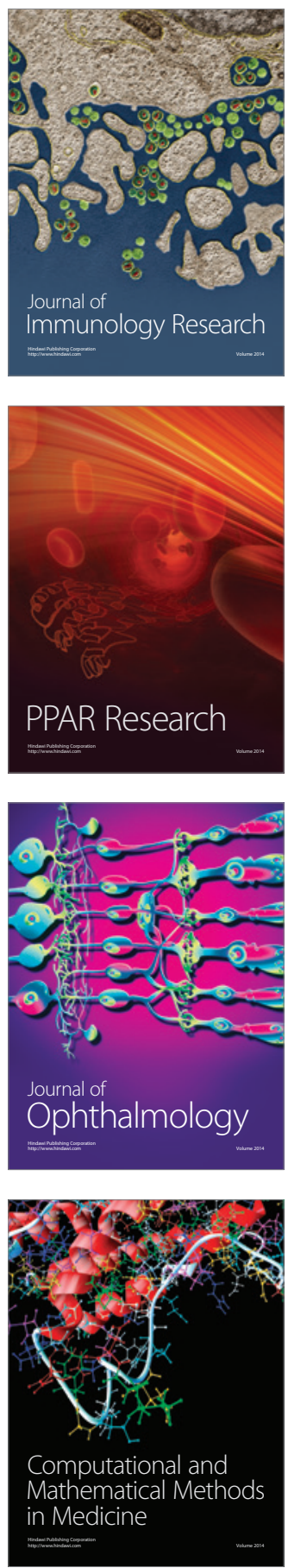

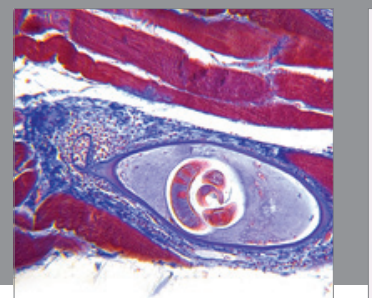

Gastroenterology

Research and Practice
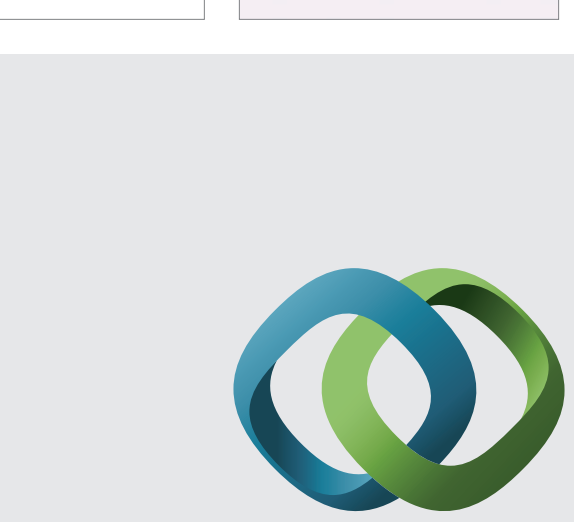

\section{Hindawi}

Submit your manuscripts at

http://www.hindawi.com
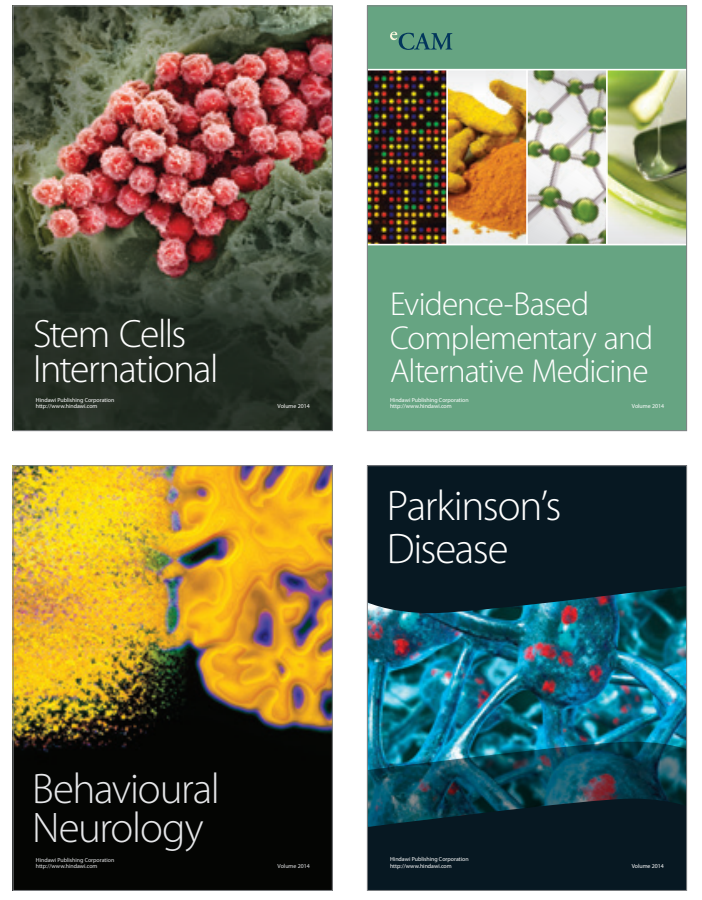
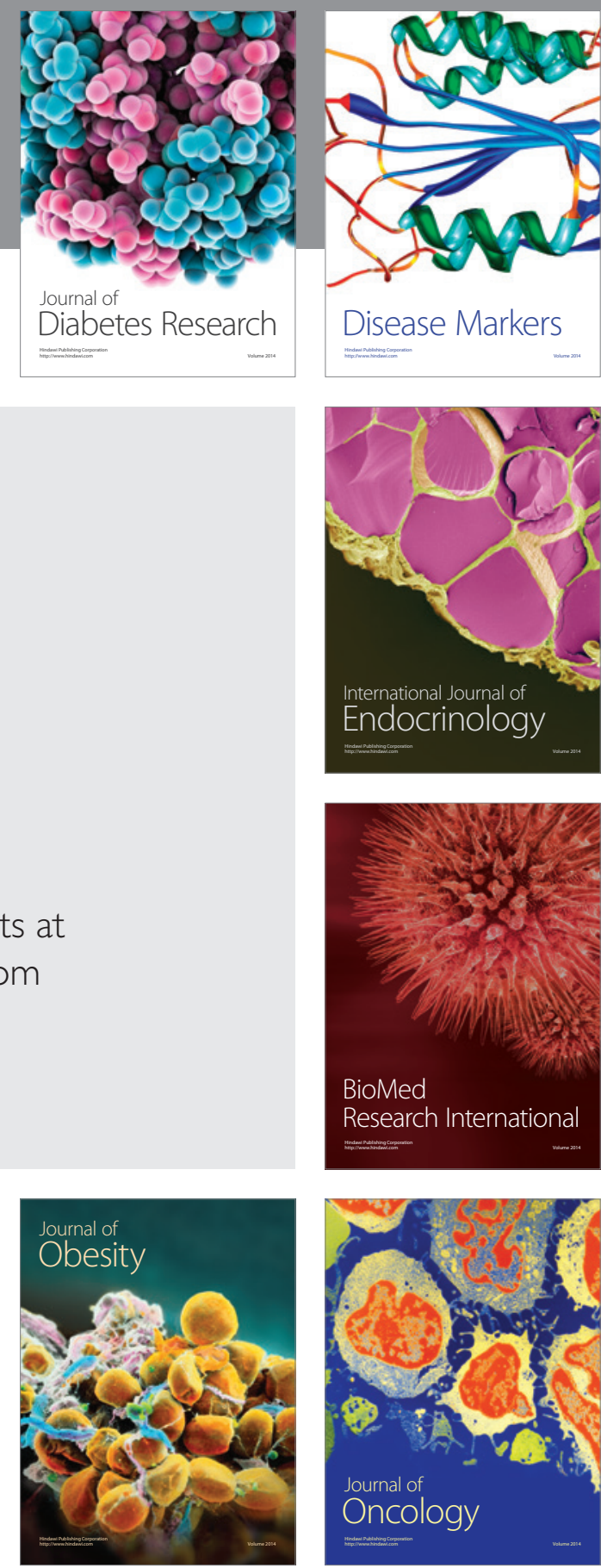

Disease Markers
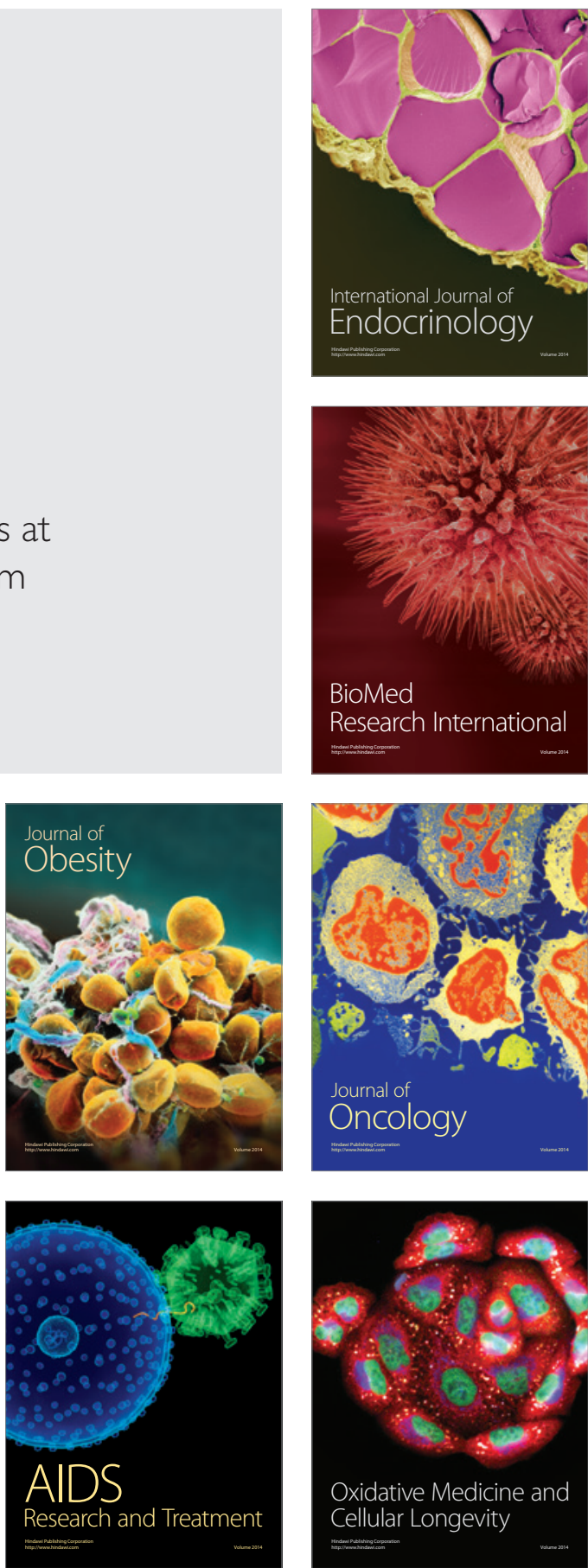